\title{
Performance Analysis for Single-fed ESPAR in the Presence of Impedance Errors and Imperfect CSI
}

\author{
Ang Li and Christos Masouros \\ Dept. of Electronic and Electrical Eng., University College London, London, UK \\ Email: ang.li.14@ucl.ac.uk, chris.masouros@ieee.org
}

\begin{abstract}
Existing MIMO precoding techniques assume conventional antenna arrays with multiple radio-frequency (RF) chains each connected to a different antenna. Towards small portable devices and base stations, single-fed compact arrays, also known as electronically steerable parasitic antenna radiators (ESPAR) have recently emerged as a new antenna structure that requires only a single $\mathrm{RF}$ chain. In this paper, we study the ESPAR based antenna arrays and explore linear precoding schemes for ESPAR antennas. The closed-form expression for the computation of the tunable loads and the feeding voltage is firstly shown and the impact of impedance errors and imperfect CSI on the performance is also investigated analytically. It will be shown that the impedance errors will act as an additional noise source that is independent of the SNR and thus result in an error floor at high SNR. We further study the energy efficiency of both conventional MIMO and ESPAR-based MIMO systems. Simulation results validate our analysis and show that ESPAR without impedance errors can achieve a similar performance to conventional antenna arrays and a higher energy efficiency, while the performance degradation due to impedance errors motivates the design of robust precoding schemes.
\end{abstract}

Index Terms-MIMO, single-fed ESPAR, impedance errors, imperfect CSI, precoding, energy efficiency

\section{INTRODUCTION}

Multiple-input-multiple-output (MIMO) systems have been widely acknowledged as a promising technology that offers significant spectral efficiency compared with single-inputsingle-output (SISO) systems and have become an indispensable part of modern wireless communication standards. In recent MIMO communications, precoding techniques have been widely studied due to the need for power and cost efficient UE devices. Among all precoding schemes, linear precoding techniques based on channel inversion (CI) in [1] offers the least complexity, but the performance of channel inversion is far from optimum. Regularized channel inversion (RCI) proposed in [2] and correlation rotation (CR) in [3] can provide further gains with respect to CI. Nevertheless, the transmission rate offered by them is still far from the theoretical channel capacity. Therefore, a number of non-linear vector perturbation (VP) precoding techniques have been proposed to further increase the transmission rates [4]-[7]. Despite the rate benefits these schemes offer, VP-based methods developed so far are complex as they require sophisticated sphere-search algorithms that make them still impractical in the present state. On the other hand, massive MIMO systems where a large number of antenna elements are employed have become a candidate technique for future $5 \mathrm{G}$ communications and therefore become a popular research topic [8]-[13]. In massive MIMO, the computational complexity for non-linear precoding will be extremely high and CI based linear precoding scheme are proved to achieve optimal capacity performance. Therefore, the advantages of linear precoding schemes and their future potential make them more appropriate for contemporary MIMO systems and future communication systems. As a result of the above, linear precoding schemes will be the focus of this paper.

Conventional antenna arrays usually operate with multiple radio-frequency (RF) chains each connected to a different antenna element. However, for lightweight and small batterypowered devices with strict size constraints, the hardware burden and power consumption induced by multiple RF chains makes them impractical. Towards this direction, an alternative single-fed compact antenna array, also known as ESPAR has emerged as a research focus [14][15]. Different from conventional MIMO where each RF chain is fed with a voltage source, in ESPAR the voltage is only fed at the sole active antenna element and therefore only single RF chain is needed, while the currents at all adjacent parasitics are induced by the strong mutual coupling effects between ESPAR antennas, enabling the parasitic antennas to radiate. Therefore, by empolying ESPAR antennas, the hardware complexity and power consumption can be well alleviated as only one RF chain and one power amplifier are needed, which meets the requirement of energy efficient communication for future wireless industry. Due to the benefits of ESPAR mentioned above, ESPARs have received research attention recently as a promising candidate for future communication systems. In [16]-[18], the ESPAR is proposed for single-RF MIMO systems where spatial multiplexing is considered. In [19], a loading scheme is proposed to support the multiplexing of two 16-QAM signals for ESPAR. In [20], the precoding schemes for ESPAR are discussed and a design guideline is given with closed form of the required feeding voltage and corresponding load values. However, specific precoding scheme was not introduced. A new signal model for ESPAR MIMO systems is introduced in [21] where the currents at the ports of the transmitting array are considered as the input to the system.

In this paper, the precoding scheme for ESPAR-based MIMO systems is studied, which is motivated by the new signal model in [22]. We focus particularly on channel inversion linear precoding technique as it is less complex and more practical. The closed-form expression for the exact 
computation of the tunable loads and the feeding voltage is firstly given based on the derivation in [20]. We further study the impact of the impedance errors and imperfect CSI caused by realistic hardware implementations and analytically derive the system performance in the presence of these two effects. It will be shown that the existence of the impedance error leads to an error floor at high SNR. Moreover, We investigate the performance of ESPAR-based system in terms of energy efficiency. It will be shown in the simulation results that ESPAR-based MIMO system is more favorable in terms of energy efficiency than conventional MIMO system as only single RF chain is needed.

Notations: $E(\cdot),(\cdot)^{T},(\cdot)^{H},(\cdot)^{-1},(\cdot)^{\dagger}$ and $\operatorname{tr}()$ denote expectation, transpose, conjugate transpose, inverse, MoorePenrose inverse and trace of a matrix respectively. $\|\cdot\|$ denotes the Frobenius norm, $\mathbf{I}_{n}$ is the $n \times n$ identity matrix and $\mathbf{0}$ denotes zero matrix or vector. $\mathbf{C}^{n \times n}$ represents $n \times n$ matrix in the complex set and $\mathbf{R}(k, u)$ denotes the element of the $k$ th-row and $u$ th-column in $\mathbf{R}$.

\section{SySTEM MOdeL}

In this section, the considered signal model which captures the features of ESPAR antennas is given, followed by the MIMO systems and the channel model we consider.

\section{A. Signal Model}

We first consider the signal model of conventional antenna array, and then extend to single-fed ESPAR antennas. Assume that a transmitter is equipped with $N_{t}$ antennas, and antenna $k\left(k \in\left[1, N_{t}\right]\right)$ is fed by a source with the complex voltage $v_{k}$ and output impedance $Z_{k}^{\text {output }}$. We denote the mutual impedance matrix of the transmit array which is decided by the antenna spacing and placement as $\mathbf{Z}_{T} \in C^{N_{t} \times N_{t}}$. Then, according to the generalized Ohm's law, the complex current vector at each antenna is given as [19]

$$
\mathbf{i}=\left(\mathbf{Z}_{G}+\mathbf{Z}_{T}\right)^{-1} \cdot \mathbf{v}_{T}=\overline{\mathbf{D}}_{T}^{-1} \mathbf{v}_{T}
$$

where $\mathbf{i}=\left[i_{1}, i_{2}, \ldots, i_{N_{t}}\right]^{T}$ denotes the current vector and $\mathbf{Z}_{G} \in C^{N_{t} \times N_{t}}$ is the diagonal matrix with the output impedance of each antenna $Z_{k}^{\text {output }}$ in the main diagonal and $\mathbf{v}_{T}=\left[v_{1}, v_{2}, \ldots, v_{N_{t}}\right]^{T}$ is the $N_{t} \times 1$ voltage vector. $\overline{\mathbf{D}}_{T}$ is the effective antenna coupling matrix.

When a transmitter is equipped with a single-fed ESPAR array, only the central active element is fed with voltage $v_{s}$, while the remaining $N_{t}-1$ elements are parasitic and excited passively. Therefore, for $\forall k \in\left[2, N_{t}\right], v_{k}=0$. Following a similar derivation, the currents in each antenna port can be expressed as

$$
\mathbf{i}=\left(\mathbf{X}+\mathbf{Z}_{T}\right)^{-1} \cdot\left[v_{s}, 0,0, \ldots, 0\right]^{T}=\mathbf{D}_{T}^{-1} \mathbf{v}_{s}
$$

where $\mathbf{X}=\operatorname{diag}\left(R_{0}, x_{1}, \ldots, x_{N_{t}-1}\right)$ is the diagonal matrix whose diagonal elements consist of the output impedance of the active element $R_{0}$ (which usually equals $50 \Omega$ ) and the tunable loads for each parasitic antennas, denoted as $x_{i}$, $i \in\left[1, N_{t}-1\right]$. For ESPAR, the effective antenna coupling matrix $\mathbf{D}_{T}$ can be controlled by tuning the loads of parasitic antennas, and therefore the currents at each antenna port can be controlled accordingly.

Motivated by the signal model in [21], here we consider the currents at the ports of the transmitting array as the input to the system and using (1) and (2) for conventional antenna array and single-fed ESPAR array respectively, a general system equation can be expressed as

$$
\mathbf{y}=\mathbf{H i}+\mathbf{w}
$$

where $\mathbf{H} \in C^{K \times N_{t}}$ is the complex channel matrix relating the input currents at the transmitter with the output open-circuit voltage vector $\mathbf{y} \in C^{K \times 1}$ at the receiver side. $K$ is the number of users and $\mathbf{w} \in C^{K \times 1}$ is the additive white Gaussian noise (AWGN) with covariance matrix $\sigma^{2} \mathbf{I}_{K}$.

\section{B. Channel Model}

We consider a multiuser MIMO downlink system where the base station communicates with $K$ users simultaneously. The base station is equipped with $N_{t}$ antennas and $K \leq N_{t}$. We assume that the base station processes the transmit symbols $\mathbf{s}$ with a precoding matrix $\mathbf{F}$ before transmission and by setting $\mathbf{i}=f \cdot \mathbf{F s}$, the open-circuit voltage vector at the receiver $k$ can be mathematically expressed as

$$
\mathbf{y}=f \cdot \mathbf{H F} \mathbf{s}+\mathbf{w}
$$

For the purposes of the analysis below, full knowledge of channel state information (CSI) is assumed at the base station, which is a common assumption for precoding schemes. However, we also investigate scenarios with imperfect CSI in the following sections. When the transmitter is equipped with ESPAR antennas, the correlation effect among antennas cannot be ignored as the spacing between antennas is small and ESPAR also operates based on strong mutual coupling between antennas. Therefore, the channel is modeled as

$$
\mathbf{H}=\mathbf{H}_{G} \mathbf{R}_{T}
$$

where the elements of $\mathbf{H}_{G} \sim \mathcal{C N}(0,1)$. $\mathbf{R}_{T}$ represents the transmit-side spatial correlation matrix and for uniform linear arrays (ULAs) its elements can be modeled as $\mathbf{R}_{T}(k, j)=$ $e^{j \cdot 2 \pi \cdot|k-j| \cdot d}$, where $d$ denotes the antenna spacing. It is worth noting that in non-ideal antenna assumption, besides the correlation effect, there also exists mutual coupling effect that should be considered. In EPSAR based array, as ESPAR operates and radiates based on the strong mutual coupling effects between antenna elements, the mutual coupling effect has already been taken into account by the mutual impedance matrix when calculating the currents, and is therefore not shown explicitly in the channel model. We assume that the receivers are equipped with ideal uncoupled and uncorrelated antennas and therefore after the signals are scaled back, only the demodulation stage is required at the receivers.

\section{Channel Inversion Precoding ANd Extension TO ESPAR}

In this section, the channel inversion precoding is first introduced, followed by the extension of the precoding scheme to ESPAR-based MIMO system. 


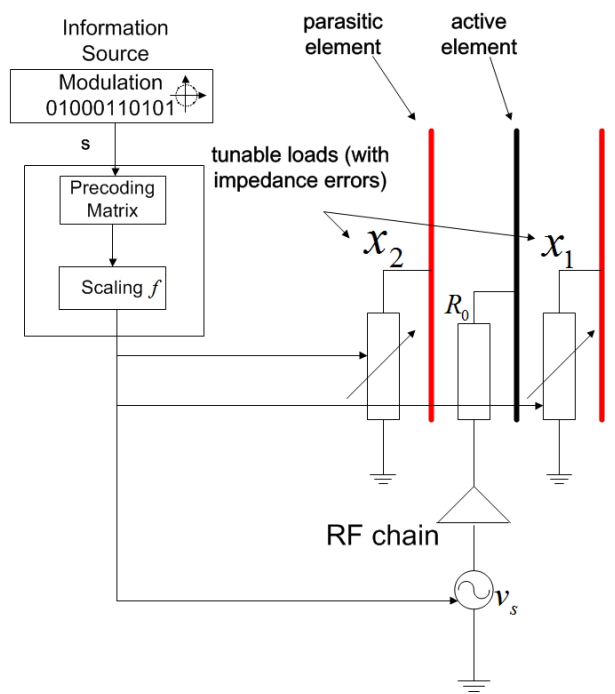

Fig. 1. ESPAR with one active element and parasitic elements

\section{A. Channel Inversion (CI) Precoding}

Based on the previous signal model, the currents are used as the input signals in ESPAR-based MIMO systems, and therefore for CI precoding, the currents can be expressed as

$$
\mathbf{i}=f \cdot \mathbf{H}^{H} \cdot\left(\mathbf{H} \cdot \mathbf{H}^{H}\right)^{-1} \cdot \mathbf{s}
$$

where $\mathbf{s}=\left[s_{1}, s_{2}, \ldots, s_{N_{t}}\right]^{T}$ is the $N_{t} \times 1$ symbol vectors. $f$ is the scaling factor that ensures $E\left(\|\mathbf{i}\|^{2}\right)=1$ and is given by $f=\sqrt{1 / \operatorname{tr}\left[\left(\mathbf{H} \cdot \mathbf{H}^{H}\right)^{-1}\right]}$. At the receiver, the signals are scaled back before demodulation, expressed as

$$
\mathbf{r}=\frac{1}{f} \mathbf{y}=\frac{1}{f}(\mathbf{H F} \mathbf{s}+\mathbf{w})=\mathbf{s}+\frac{\mathbf{w}}{f}
$$

\section{B. Computation of Loads and Feeding Voltage}

Fig. 1 shows the block diagram of ESPAR-based MIMO system where only the central active antenna element is connected to a voltage source with the RF chain. In ESPAR, the tunable loads are used to control the effective antenna coupling matrix, and therefore control the currents. By expanding (2), the feeding voltage and the value of each tunable loads can be obtained as [20]

$$
\begin{aligned}
& v_{s}=\sum_{m=1}^{N_{t}} Z_{1, m} \cdot i_{m}+R_{s} \cdot i_{1} \\
& x_{k}=-\frac{1}{i_{k+1}} \cdot \sum_{m=1}^{N_{t}} Z_{k+1, m} \cdot i_{m}
\end{aligned}
$$

As mentioned earlier, the currents are dependent on the precoding schemes and the symbols that are known before transmission. Therefore, by setting the feeding voltage and the loads as calculated, the ESPAR can radiate signals as conventional MIMO.

\section{Performance Analysis in the Presence of IMPEDANCE ERRORS AND IMPERFECT CSI}

\section{A. Imperfect CSI Model and Impedance Error Model}

In order to investigate the effect of errors in CSI in realistic systems, in this paper we assume that the system is operating in TDD mode and the CSI $\hat{\mathbf{H}}$ is directly measured at the transmitter using uplink-downlink reciprocity and is subject to noise errors [22]. Therefore, the errors are modelled as inversely proportional to the transmit SNR, expressed as

$$
\hat{\mathbf{H}}=\mathbf{H}+\mathbf{E}
$$

with $\mathbf{E} \sim \mathcal{C N}\left(0, \eta \cdot \mathbf{I}_{N_{t}}\right)$, statistically independent to $\mathbf{H}$, where $\eta=\alpha \cdot\left(\frac{P}{\sigma^{2}}\right)^{-1}$ with $\alpha$ being the inversely proportional coefficient. As we assume the equal transmit SNR for each user, therefore we assume the channel error coefficient for each user remains the same. Then, as $\mathbf{H}$ and $\mathbf{E}$ are statistically independent random Gaussian, $\mathbf{H}$ and $\hat{\mathbf{H}}$ are joint Gaussian. Therefore, $\mathbf{H}$ follows a Gaussian distribution with mean $\frac{\hat{\mathbf{H}}}{1+\eta}$ and variance $\frac{\eta}{1+\eta}$, given by [23]

$$
\mathbf{H}=\frac{1}{1+\eta} \cdot \hat{\mathbf{H}}+\mathbf{Q}
$$

where $\mathbf{Q} \sim \mathcal{C N}\left(0, \frac{\eta}{1+\eta} \cdot \mathbf{I}_{N_{t}}\right)$.

Due to the realistic hardware implementations, there may exist impedance errors for the tunable loads, which will degrade the system performance. In this paper, we assume the value of impedance errors is statistically independent to the impedance value and is modeled as a complex Gaussian variable [25][26], expressed as

$$
\hat{x}_{k}=x_{k}+\varepsilon_{k}
$$

where for each $k, \varepsilon_{k} \sim \mathcal{C N}(0, \delta)$ with $\delta$ being the variance.

\section{B. Performance Analysis - Impedance Errors, Perfect CSI}

First we focus on the scenarios with only the impedance errors. Consider each tunable load value with error $\varepsilon_{k}$ independently, then (2) can be rewritten as

$$
\hat{\mathbf{i}}=\left(\mathbf{X}+\mathbf{E}_{\varepsilon}+\mathbf{Z}_{T}\right)^{-1} \cdot\left[v_{s}, 0, \ldots, 0\right]^{T}=\left(\mathbf{D}_{T}+\mathbf{E}_{\varepsilon}\right)^{-1} \mathbf{v}_{s}
$$

where $\mathbf{E}_{\varepsilon}=\operatorname{diag}\left(0, \varepsilon_{1}, \ldots, \varepsilon_{N_{t}-1}\right)$ is the impedance error matrix. In cases with impedance errors, the feeding voltage remains the same as we are not aware of the errors, and therefore

$$
\left(\mathbf{D}_{T}+\mathbf{E}_{\varepsilon}\right) \cdot \hat{\mathbf{i}}=\mathbf{D}_{T} \cdot \mathbf{i}
$$

As $\mathbf{i}$ is a column vector, the currents with impedance errors can be further transformed into

$$
\begin{aligned}
\hat{\mathbf{i}} & =\left(\mathbf{D}_{T}+\mathbf{E}_{\varepsilon}\right)^{-1} \cdot \mathbf{D}_{T} \cdot \mathbf{i} \\
& =\left(\mathbf{D}_{T}+\mathbf{E}_{\varepsilon}\right)^{-1} \cdot\left(\mathbf{D}_{T}+\mathbf{E}_{\varepsilon}-\mathbf{E}_{\varepsilon}\right) \cdot \mathbf{i} \\
& =\mathbf{i}-\left(\mathbf{D}_{T}+\mathbf{E}_{\varepsilon}\right)^{-1} \cdot \mathbf{E}_{\varepsilon} \cdot \mathbf{i}
\end{aligned}
$$

Then, the received signals with impedance errors can be obtained by substituting (15) into (3), expressed as

$$
\begin{aligned}
\mathbf{y} & =\mathbf{H} \cdot\left[\mathbf{i}-\left(\mathbf{D}_{T}+\mathbf{E}_{\varepsilon}\right)^{-\mathbf{1}} \mathbf{E}_{\varepsilon} \mathbf{i}\right]+\mathbf{w} \\
& =\mathbf{H i}-\mathbf{H}\left(\mathbf{D}_{T}+\mathbf{E}_{\varepsilon}\right)^{-\mathbf{1}} \mathbf{E}_{\varepsilon} \mathbf{i}+\mathbf{w}
\end{aligned}
$$


By substituting $\mathbf{i}=f \cdot \mathbf{H}^{H} \cdot\left(\mathbf{H} \cdot \mathbf{H}^{H}\right)^{-1} \cdot \mathbf{s}$, the received signals can be further transformed into

$$
\mathbf{y}=f \cdot \mathbf{s}-\mathbf{H}\left(\mathbf{D}_{T}+\mathbf{E}_{\varepsilon}\right)^{-\mathbf{1}} \mathbf{E}_{\varepsilon} \mathbf{i}+\mathbf{w}
$$

As can be seen from (17), the second term $\mathbf{H}\left(\mathbf{D}_{T}+\mathbf{E}_{\varepsilon}\right)^{-1} \mathbf{E}_{\varepsilon} \mathbf{i}$ acts as an additional noise term. We then define the equivalent noise term

$$
\hat{\mathbf{w}} \triangleq-\mathbf{H}\left(\mathbf{D}_{T}+\mathbf{E}_{\varepsilon}\right)^{-\mathbf{1}} \mathbf{E}_{\varepsilon} \mathbf{i}+\mathbf{w}
$$

In general, as we must ensure a positive input impedance, $Z_{11}$ is usually chosen with a large value, and $\mathbf{E}_{\varepsilon}$ will be very small compared to $\mathbf{D}_{T}=\mathbf{X}+\mathbf{Z}_{T}$. Therefore, based on the "small errors" assumption in [27] and for analytical tractability we approximate $\tilde{\mathbf{w}}$ as

$$
\hat{\mathbf{w}} \approx-\mathbf{H D}_{T}^{-1} \mathbf{E}_{\varepsilon} \mathbf{i}+\mathbf{w}
$$

By applying a similar derivation in [15] [16] and noting that $\mathbf{i}$ is normalized, $\hat{\mathbf{w}} \sim \mathcal{C N}\left(0, \hat{v}_{\varepsilon}\right)$ where the variance $\hat{v}_{\varepsilon}$ is given by

$$
\hat{v}_{\varepsilon}=\zeta^{2} \cdot \delta+\sigma^{2}
$$

where $\zeta=\left\|\mathbf{D}_{T}^{-1}\right\|$. Then, based on this the resulting received SNR is then obtained as

$$
\gamma_{i}=f^{2} \cdot \frac{\left\|s_{i}\right\|^{2}}{\left(\zeta^{2} \cdot \delta+\sigma^{2}\right)}
$$

It is observed that the first term of the noise is irrelevant to SNR, and therefore at high SNR regime when the second term becomes negligible while the first term persists, an error floor could be observed, which will be validated by the simulation results. Then, the achievable sum rate could be obtained as

$$
R=\sum_{i=1}^{K} \log _{2}\left(1+\gamma_{i}\right)
$$

\section{Performance Analysis - Impedance Errors, Imperfect CSI}

Let us proceed to the study of the performance of ESPARbased MIMO system under scenarios with imperfect CSI and impedance errors. In such cases, by substituting (11) and (15) into (3), the received signals can be obtained as

$$
\begin{aligned}
\mathbf{y} & =\mathbf{H} \cdot \hat{\mathbf{i}}+\mathbf{w} \\
& =\left(\frac{1}{1+\eta} \hat{\mathbf{H}}+\mathbf{Q}\right) \mathbf{i}-\mathbf{H}\left(\mathbf{D}_{T}+\mathbf{E}_{\varepsilon}\right)^{-\mathbf{1}} \mathbf{E}_{\varepsilon} \mathbf{i}+\mathbf{w} \\
& =\frac{1}{1+\eta} \hat{\mathbf{H}} \mathbf{i}+\mathbf{Q} \mathbf{i}-\mathbf{H}\left(\mathbf{D}_{T}+\mathbf{E}_{\varepsilon}\right)^{-\mathbf{1}} \mathbf{E}_{\varepsilon} \mathbf{i}+\mathbf{w}
\end{aligned}
$$

In cases with imperfect CSI, the currents for CI precoding is $\mathbf{i}=\hat{f} \cdot \hat{\mathbf{H}}^{H}\left(\hat{\mathbf{H}} \cdot \hat{\mathbf{H}}^{H}\right)^{-1} \mathbf{s}$, and the received signals can be further transformed into

$$
\mathbf{y}=\frac{\hat{f}}{1+\eta} \cdot \mathbf{s}+\mathbf{Q i}-\mathbf{H}\left(\mathbf{D}_{T}+\mathbf{E}_{\varepsilon}\right)^{-\mathbf{1}} \mathbf{E}_{\varepsilon} \mathbf{i}+\mathbf{w}
$$

The second term and third term in (24) will act as the additional noise. Similarly, the equivalent noise for imperfect CSI and impedance errors can be approximated as

$$
\hat{\mathbf{w}} \triangleq \mathbf{Q} \mathbf{i}-\mathbf{H D}_{T}^{-1} \mathbf{E}_{\varepsilon} \mathbf{i}+\mathbf{w}
$$

Noting that $\mathbf{i}$ is normalized and $\mathbf{Q} \sim \mathcal{C N}\left(0, \frac{\eta}{1+\eta} \cdot \mathbf{I}_{N_{t}}\right), \hat{\mathbf{w}} \sim$ $\mathcal{C N}\left(0, \hat{v}_{\varepsilon}\right)$ and $\hat{v}_{\varepsilon}$ is given as

$$
\hat{v}_{\varepsilon}=\frac{\eta}{1+\eta}+\zeta^{2} \cdot \delta+\sigma^{2}
$$

It is shown in [20] that the signals must scale back by $\frac{1+\eta}{\hat{f}}$ in the presence of imperfect CSI, and therefore the signals after scaling back can be calculated as

$$
\mathbf{r}=\frac{1+\eta}{\hat{f}} \mathbf{y}=\mathbf{s}+\frac{1+\eta}{\hat{f}} \hat{\mathbf{w}}
$$

Therefore, the resulting received SNR can be calculated as

$$
\hat{\gamma}_{i}=\frac{\hat{f}^{2}}{(1+\eta)^{2}} \cdot \frac{\left\|s_{i}\right\|^{2}}{\frac{\eta}{1+\eta}+\zeta^{2} \cdot \delta+\sigma^{2}}
$$

Then, the achievable sum rate could be obtained as

$$
\hat{R}=\sum_{i=1}^{K} \log _{2}\left(1+\hat{\gamma}_{i}\right)
$$

\section{Probability of Error}

In order to validate the above analysis, we introduce the probability of bit error for QPSK in flat fading, as assumed in this paper, with respect to the received SNR, which is expressed as [24]

$$
P_{e}=\frac{1}{2}\left(1-\sqrt{\frac{\beta}{\beta+1}}\right)
$$

For scenarios with impedance errors under perfect CSI, $\beta$ is the energy-to-noise ratio per bit $\left(E_{b} N_{0}\right)$ and can be obtained based on (21) as

$$
\beta=\frac{1}{2 \cdot K \cdot\left(\zeta^{2} \cdot \delta+\sigma^{2}\right)}
$$

For scenarios with impedance errors under imperfect CSI, the analytical $\beta$ could be similarly obtained based on (28) and expressed as

$$
\beta=\frac{1}{2 \cdot K \cdot\left(\frac{\eta}{1+\eta}+\zeta^{2} \cdot \delta+\sigma^{2}\right)}
$$

The analytical probability of bit error calculation is then compared to the simulation results in the following section.

\section{ENERGY EFFICIENCY}

To evaluate the usefulness of the ESPAR-based MIMO system, we will investigate the tradeoff between performance and complexity by means of the resulting energy efficiency. In this section,we define the energy efficiency of the communication system as the sum rate per total transmit power consumed, shown as [6][7]

$$
E E=\frac{R}{N_{R F} \cdot P_{A C}+P_{P A}+P_{0}}
$$

where $R$ is defined by (22) and (29), $P_{A C}$ is the constant radio frequency $(\mathrm{RF})$ circuit power consumption per antenna element, $P_{P A}=(\mu / \varepsilon-1) \cdot P$ denotes the total power 


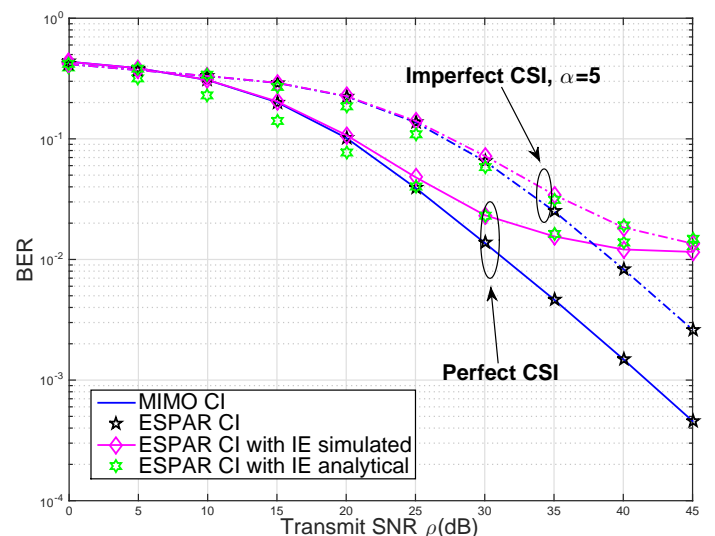

Fig. 2. BER performance of conventional MIMO and ESPAR-based MIMO, $N_{t}=K=2, \delta=1.5$, QPSK

consumption for the power amplifier where $\mu=3 \frac{\sqrt{M}-1}{\sqrt{M}+1}$ is the modulation dependent peak to average power ratio (PAPR) for M-QAM modulation and $\varepsilon$ is the power amplifier efficiency [5]. $P_{0}$ is the baseband signal processing power consumption which is assumed fixed. The values of each parameter in the following simulations are as follows: $\varepsilon=0.35, P_{A C}=$ $33 \mathrm{dBm}$ and $P_{0}=40 \mathrm{dBm}$. It is worth noting that $N_{R F}=N_{t}$ for conventional MIMO and $N_{R F}=1$ for the single-fed ESPAR implementation.

\section{NUMERICAL RESULTS}

To evaluate the performance of ESPAR-based MIMO systems, in this section numerical results based on Monte Carlo simulations of MIMO CI and ESPAR-based CI are presented. QPSK modulation is applied in the simulations. We assume $N_{t}=K=2$, i.e. ESPAR has one active element with one parasitic element. For simplicity, we assume $P=1$ and the variance of the impedance errors $\delta=1.5$ unless otherwise stated. The spacing between ESPAR antennas is assumed as $d=\lambda / 4$ and the mutual coupling matrix is therefore obtained as $Z_{T}=\left[\begin{array}{cc}465.4-659.5 i & -24.06+34.93 i \\ -24.06+34.93 i & 21-157.2 i\end{array}\right]$ to ensure a positive real part of the input impedance. For clarity the following abbreviations are used: "MIMO CI" for MIMO CI,"ESPAR CI" for ESPAR CI without impedance errors, and "ESPAR with IE" for ESPAR CI with impedance errors.

Firstly, to verify the performance of ESPAR-based MIMO system, Fig. 2 compares the bit error rate (BER) performance of conventional MIMO system and ESPAR-based MIMO system. As can be seen, in the cases where no impedance errors, ESPAR-based MIMO system can achieve the same performance as conventional MIMO under the signal model in this paper. It is also observed that in the presence of channel errors, the system suffers a performance degradation due to the inaccurate channel state information. In the cases where there exist impedance errors, an error floor could be observed at high SNR and the simulated results are well matched to the analytical results, which validates our analysis above.

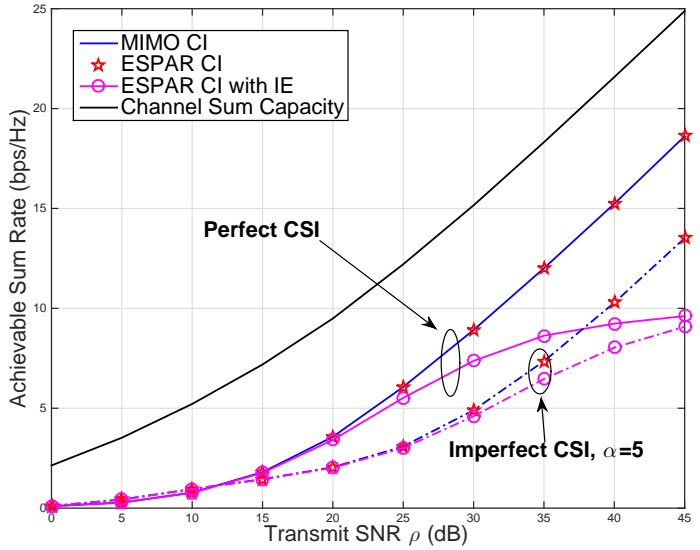

Fig. 3. Achievable sum rate of MIMO and ESPAR-based MIMO, $N_{t}=K=$ $2, \delta=1.5$, QPSK

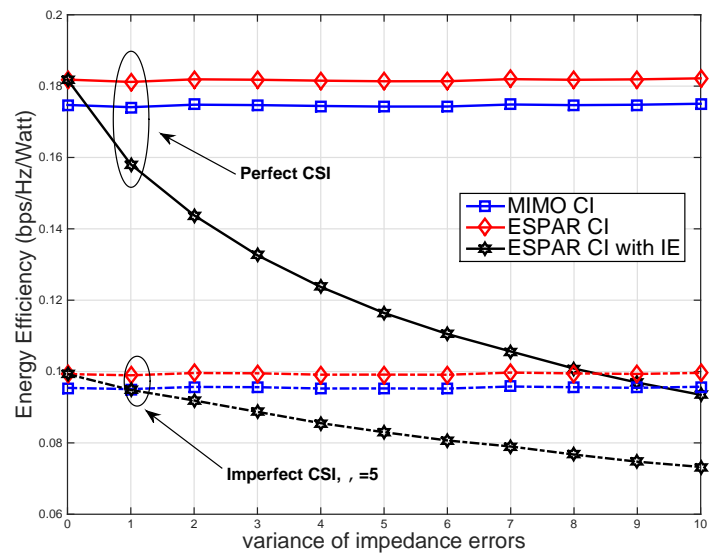

Fig. 4. Energy efficiency, $P=43 \mathrm{dBm}, \mathrm{SNR}=30 \mathrm{~dB}, N_{t}=K=2, \delta=1.5$, QPSK

Fig. 3 compares the achievable sum rate of convention MIMO and ESPAR-based MIMO system where the channel sum capacity is given as

$$
C=E\left\{\sup _{\mathbf{D} \in A} \log _{2}\left[\operatorname{det}\left(\mathbf{I}+\frac{1}{\sigma^{2}} \mathbf{H}^{H} \mathbf{D H}\right)\right]\right\}
$$

where sup denotes the supremum function and $A$ is the set of diagonal $K \times K$ matrices with nonnegative elements to ensure $\operatorname{tr}(\mathbf{D})=1$. When equal transmit power allocation is assumed, $\mathbf{D}=(1 / K) \cdot \mathbf{I}$. As can be seen, ESPAR-based MIMO without impedance errors achieves the same performance to conventional MIMO for CI precoding. For ESPAR-based MIMO with impedance errors, a rate floor at high SNR could be seen because of the additional noise introduced by the impedance errors which is irrelevant to the transmit SNR, as validated by the previous analysis. Moreover, it is observed that both MIMO systems under perfect CSI achieve a higher rate performance than in the presence of imperfect CSI 
Fig. 4 shows the energy efficiency of two MIMO system with respect to the increasing variance of the impedance error. First of all, it can be seen that the system under perfect CSI achieves a higher energy efficiency performance than that under impefect CSI for both conventional MIMO system and ESPAR-based MIMO system. Then, it is observed that ESPAR-based MIMO system without impedance errors achieves a higher energy efficiency performance than conventional MIMO system due to the reduced power consumption as illustrated by (33). It is also observed that in the presence of the impedance errors, the system performance of ESPARbased MIMO with impedance errors suffers a degradation because of the degradation in the sum rate as shown in Fig. 3. Moreover, with the increasing of the impedance error variance, the system performance degradation becomes more severe.

\section{CONCLUSION}

In this paper the linear precoding techniques for ESPARbased MIMO systems is studied. Firstly, the computation of the tunable loads and the feeding voltage for channel inversion precoding schemes is given. Then, we analytically derive the received SNR in the presence of impedance errors and imperfect CSI for ESPAR-based MIMO system. It is shown that impedance errors introduces an additional noise and will result in an error floor at high SNR regime. Furthermore, we study the energy efficiency performance of two MIMO systems. Simulation results validate our analysis and show that without impedance errors, ESPAR-based MIMO system achieves a similar performance to conventional MIMO while it suffers a performance degradation with impedance errors. Moreover, it is also shown that ESPAR-based MIMO system could achieve a higher performance in energy efficiency due to the reduced power consumption. Since the impedance errors will severely degrade the ESPAR-based MIMO systems, the robust precoding schemes that can alleviate this effect would be the future research focus.

\section{ACKNOWLEDGEMENT}

This work was supported by the Royal Academy of Engineering, UK and the Engineering and Physical Sciences Research Council (EPSRC) project EP/M014150/1.

\section{REFERENCES}

[1] D. Senaratne, C. Tellambura, and H. A. Suraweera, "Performance Analysis of MIMO Channel Inversion in Rayleigh Fading," IEEE Trans. Veh. Tech., vol. 61, no. 3, pp. 1188-1196, 2012.

[2] C. B. Peel, B. M. Hochwald, and A. L. Swindlehurst, "A vector-perturbation technique for near-capacity multiantenna multiuser communication-part I: channel inversion and regularization," IEEE Trans. Commun., vol. 53, no. 1, pp. 195-202, Jan 2005.

[3] C. Masouros, "Correlation Rotation Linear Precoding for MIMO Broadcast Communications," IEEE Trans. Sig. Process., vol. 59, no. 1, pp. 252-262, Jan. 2011.

[4] C. B. Peel, B. M. Hochwald, and A. L. Swindlehurst, "A vector-perturbation technique for near-capacity multiantenna multiuser communication-part II: perturbation," IEEE Trans. Commun., vol. 53, no. 3, pp. 537-544, Mar. 2005.

[5] C. Masouros, M. Sellathurai, and T. Ratnarajah, "Computationally efficient vector perturbation precoding using thresholded optimization," IEEE Trans. Commun., vol. 61, no. 5, pp. 1880-1890, May 2013
[6] C. Masouros, M. Sellathurai, and T. Ratnarajah, "Maximizaing energyefficiency in the vector precoded MU-MISO downlink by selective perturbation," IEEE Trans. Wireless Commun., vol. 13, no. 9, pp. 49744984, Sep. 2014.

[7] A. Li, and C. Masouros, "A Constellation Scaling Approach to Vector Perturbation for Adaptive Modulation in MU-MIMO," IEEE Wireless Commun. Lett., vol. 4, no. 3, pp. 289-292, June 2015.

[8] E. Larsson, O. Edfors, F. Tufvesson, and T. Marzetta, "Massive MIMO for next generation wireless systems," IEEE Commun. Mag., vol. 52, no. 2, pp. 186-195, Feb. 2014.

[9] C. Masouros, M. Sellathurai, and T. Ratnarajah, "Large-Scale MIMO Transmitters in Fixed Physical Spaces: The Effect of Transmit Correlation and Mutual Coupling," IEEE Trans. Commun., vol. 61, no. 7, pp. 2794 2804, July 2013.

[10] C. Masouros, and M. Matthaiou, "Space-Constrained Massive MIMO: Hitting the Wall of Favourable Propagation," IEEE Commun. Lett., vol. 19, no. 5, pp. 771-774, May 2015.

[11] S. Biswas, C. Masouros, and T. Ratnarajah, "Performance Analysis of Large Multi-User MIMO Systems with Space-Constrained 2D Antenna Arrays," IEEE Trans. Wireless Commun., in press.

[12] A. Garcia, and C. Masouros, "Exploiting the Increasing Correlation of Space Constrained Massive MIMO for CSI Relaxation," IEEE Trans. Commun., in press.

[13] P. V. Amadori, and C. Masouros, "Interference Driven Antenna Selection for Massive Multi-User MIMO," IEEE Trans. Veh. Tech., in press.

[14] A. Kalis, A. G. Kanatas, and C. B. Papadias, "A Novel Approach to MIMO Transmission Using a Single RF Front End," in Selected Areas in Communications, IEEE Journal on, vol. 26, no. 6, pp. 972-980, August 2008.

[15] O. N. Alrabadi, C. B. Papadias, A. Kalis, and R. Prasad, "A Universal Encoding Scheme for MIMO Transmission Using a Single Active Element for PSK Modulation Schemes," in IEEE Trans. Wireless Commun., vol. 8, no. 10, pp. 5133-5143, October 2009.

[16] A. Kalis, A. Kanatas, and C. B. Papadias, Parasitic Antenna Arrays for Wireless MIMO Systems, Springer, 2014.

[17] O. N. Alrabadi, C. Divarathne, P. Tragas, A. Kalis, N. Marchetti, C. B. Papadias, and R. Prasad, "Spatial multiplexing with a single radio: Proof-of-concept experiments in an indoor environment with a $2.6 \mathrm{GHz}$ prototype," IEEE Commun. Lett., vol. 15. No. 2, pp. 178-180, Feb 2011.

[18] D. V. Thiel, and S. Smith, Switched Parasitic Antennas for Cellular Communications. Artech House, 2002.

[19] B. Han, V. I. Barousis, C. B. Papadias, A. Kalis, and R. Prasad, "MIMO over ESPAR with 16-QAM Modulation," IEEE Wireless Commun Lett., vol. 2, no. 6, pp. 687-690, Dec 2013.

[20] V. I. Barousis, and C. B. Papadias, "Arbitrary Precoding with Single-Fed Parasitic Arrays: Closed-Form Expression and Design Guidelines," IEEE Wireless Commun Lett., vol. 3, no. 2, pp. 229-232, April 2014.

[21] V. I. Barousis, R. R. Muller, and C. B. Papadias, "a new signal model for MIMO communication with compact parasitic arrays," in Proc. IEEE ISCCSP, Athens, 21-23 May 2014, pp. 109-113.

[22] C. Masouros, M. Sellathurai, and T. Ratnarajah, "Vector Perturbation Based on Symbol Scaling for Limited Feedbback MISO Downlinks," IEEE Trans. Signal Process., vol. 62, no. 3, pp. 562-571, Feb 2014.

[23] J. Maurer, J. Jalden, D. Seethaler, and G. Matz, "Vector Perturbation Precoding Revisited," IEEE Trans. Signal Process., vol. 59, no. 1, pp. 315-328, Jan 2011.

[24] G. L. Stuber, Principles of Mobile Communication. New York, 2011.

[25] S. L. Carson, M. E. Orazem, O. D. Crisalle, and L. Garcia-Rubio, "On the Error Structure of Impedance Measurements - Simulation of FRA Instrumentation", Journal of the Electrochemical Society, vol. 150, no. 10, pp. 477-490, 2003.

[26] S. L. Carson, M. E. Orazem, O. D. Crisalle, and L. Garcia-Rubio, "On the Error Structure of Impedance Measurements - Series Expansions", Journal of the Electrochemical Society, vol. 150, no. 10, pp. 501-511, 2003.

[27] F. Ciucci, T. Carraro, W. C. Chueh, and W. Lai, "Reducing Error and Measurement Time in Impedance Spectroscopy using Model Based Optimal Experimental Design”, Electrochimica Acta, vol. 56, no. 15, pp. 5416-5434, 2011.

[28] A. Li, and C. Masouros, "A Two-stage Vector Perturbation Scheme for Adaptive Modulation in Downlink MU-MIMO," IEEE Trans. Veh. Tech. DOI: 10.1109/TVT.2015.2489263, Oct. 2015. 